\title{
Findings of an effect of gender, but not handedness, on self-reported motion sickness propensity
}

\author{
Ruth E. Propper ${ }^{*}$, Frederick Bonato, Leanna Ward and Kenneth Sumner
}

\begin{abstract}
Discrepant input from vestibular and visual systems may be involved in motion sickness; individual differences in the organization of these systems may therefore give rise to individual differences in propensity to motion sickness. Non-right-handedness has been associated with altered cortical lateralization of vestibular function, such that non-right-handedness is associated with left hemisphere, and right-handedness with right hemisphere, lateralized, vestibular system. Interestingly, magnocellular visual processing, responsible for motion detection and ostensibly involved in motion sickness, has been shown to be decreased in non-right-handers. It is not known if the anomalous organization of the vestibular or magnocellular systems in non-right-handers might alter susceptibility to motion sickness. Undergraduate college students completed online versions of the Edinburgh Handedness Inventory (Oldfield, Neuropsychologia 9:97-113, 1971) and Motion Sickness Susceptibility Questionnaire - Short Form (Golding, Personality and Individual Differences 41:237-48, 2006). Data from 664 undergraduate men and women revealed no support for individual differences in handedness effects on self-reported propensity to experience motion sickness in either childhood or adulthood. Findings replicate previous work of increased motion sickness in women, compared with men. Men reported being less motion sick in adulthood compared to childhood, while women reported no differences in motion sickness between childhood and adulthood. Handedness results are discussed in the context of reported individual differences in handedness effects on memory, and gender effects in terms of social desirability. Given that the handedness groups demonstrate differences in brain organization, the null effects here may shed light on the neural mechanisms that are involved in motion sickness.
\end{abstract}

Keywords: Handedness, Motion sickness, Lateralization, Gender

Individual differences in handedness have been associated with individual differences in cognition and behavior across a range of domains. For example, individuals who are inconsistent in their hand use preference (inconsistent-handers, those who do not strongly prefer the use of one or the other hand across a variety of tasks $[\mathrm{ICH}])$, differ from those who are consistently-right-handed (consistent-right-handers, those who consistently prefer their right hand across a variety of tasks [CRH]), in free recall, recall of personal autobiographical memories, susceptibility to false memory, memory for faces, openness to persuasion, gullibility, the effectiveness of placebos, magical ideation, musical preference, sensation seeking, cognitive dissonance, sleep architecture, risk perception, and sense

\footnotetext{
* Correspondence: propperr@montclair.edu

Montclair State University, 1 Normal Avenue, Montclair, NJ 07043, USA
}

of disgust, to name just a few of the cognitive domains differing as a function of ICH versus CRH handedness (see [12], for review).

These individual differences in handedness have been proposed to reflect individual differences in cortical organization between the ICH and CRH. Specifically, ICH have a larger corpus callosum [7], increased interhemispheric interaction (see Pritchard, [12] for review), decreased structural and functional hemispheric asymmetries (e.g. [6]), and increased right hemisphere activity and access to right hemisphere processes ([12]; Propper et al., 2012). These structural and functional differences are thought to be reflected in individual differences in handedness effects in cognition and behavior.

Non-right-handedness has also been associated with altered cortical lateralization of vestibular function $[1,4]$; 
non-right-handedness is associated with left hemisphere lateralized, and right-handedness with right hemisphere lateralized, vestibular system. Interestingly, magnocellular visual processing, responsible for motion detection, is decreased in non-right-handers [15].

It has been hypothesized that one possible cause of motion sickness is the experiencing of discrepant information between the visual and vestibular systems [14]. Individual differences in the structural or functional organization of these systems might give rise to individual differences in motion sickness propensity. The current Brief Study is a preliminary investigation into individual differences in handedness effects on selfreported motion sickness.

Two lines of research suggest that ICH may have decreased motion sickness compared to CRH. First, increased asymmetry between the left and right otoliths is associated with increased motion sickness [9]. Although, to our knowledge, no examination of otolith asymmetry as a function of handedness has been conducted, given previous research indicating decreased asymmetries in nonright handers [6], it is possible that $\mathrm{ICH}$ are more symmetrical with regard to otolith organs, and may therefore be less likely to experience motion sickness.

Second, in the only published study of which we are aware that examines handedness and motion sickness, Mirabile and Teicher [8] found that individuals who reported writing only or also with their left hand (classified as left-handed) were more likely to be both inpatients at a mental institution and have intermediate levels of motion sickness compared with non-patients. Differences between overall susceptibility to motion sickness as a function of handedness were reported as significant, but it is not clear in the article in which direction these differences exist. Examination of the data within suggest that in non-patients, there may be a higher percentage of non-right-handers among those least likely to experience motion sickness, compared to right-handers. Taken together, ICH may report decreased motion sickness, compared to $\mathrm{CRH}$.

Because previous research indicates gender differences in motion sickness susceptibility, with women being more likely to self-report motion sickness [11], we also examined gender effects here.

\section{Method}

\section{Participants}

Data from 757 undergraduate students were examined. Individuals completed 2 questionnaires in order to receive 1 research credit for their Psychology class. Eighty-one individuals were excluded from analyses due to missing information, incorrectly completed questionnaires, duplicate submissions, or being age outliers (38-54). One individual was excluded as a data outlier (Final $N=675$; 534 women and 141 men; Age $M=19.67, S d=2.20$, Age Range 18-35).

\section{Materials and procedure}

The Edinburgh Handedness Inventory ([10]; EHI) and the Motion Sickness Susceptibility Questionnaire - Short Form ([5]; MSSQSF) were presented, and data recorded, via Qaultrics software (Qualtrics, Provo, UT).

In the EHI participants state whether they Always/Usually/Have No Preference for using their left or right hand for 10 common activities. Scoring is $-10 /+10,-5 /+5$ or 0 , respectively for left versus right, or no preference responses; therefore scores range on a continuum from 100 to +100 , with -100 indicating perfect consistent-lefthandedness and +100 perfect consistent-right-handedness.

The MSSQSF asks participants to report the level of their motion sickness frequency, using a likert scale ranging from 0 (never sick) to 3 (frequently felt sick), for 9 forms of movement/transportation. Experiences are rated for childhood (before age 12) and for adulthood. Motion sickness is scored for Childhood and for Adulthood separately, using the formula: Motion Sickness $=($ total sickness score calculated by adding likert choices) x (9) / (9 -number of motion/transportation types not experienced (as a child or as an adult).

Participants completed the questionnaires, via their internet connection, after providing online, anonymous consent, with order of questionnaire presentation randomized.

\section{Results}

A median split was used to define handedness groups (see [12]); those scoring at or above the absolute value of the median (EHI score $=85)$ were CRH (311 women, 60 men), and those scoring between -85 and +85 were ICH (215 women, 78 men). Because only approximately $2-3 \%$ of the population is consistently-left-handed (Lansky et al., 1988, CLH), CLH may differ neuroanatomically and behaviorally from the $\mathrm{ICH}$ and the $\mathrm{CRH}$ (see [12]), and only 11 participants scored in this range (8 women, 3 men), CLH were excluded from analyses. Final $\mathrm{N}$ analyzed $=664$ ( 526 women, 138 men).

A 2 (MSSQSF Age: Childhood versus Adulthood) $\times 2$ (Handedness: $\mathrm{CRH}$ versus ICH) $\times 2$ (Gender: Men versus Women) mixed-ANOVA revealed main effects of Gender $(\mathrm{F}(1,1)=22.13, p=<.0001)$ and MSSQSF Age

Table 1 MSSQSF mean, sd, and effect sizes, as a function of Handedness and Age

\begin{tabular}{llll}
\hline Handedness and gender & \multicolumn{2}{l}{ MSSQSF age } & \\
\cline { 2 - 3 } & Childhood & Adulthood & $\mathrm{n}$ \\
\hline ICH & $7.85(6.16)$ & $7.13(6.02)$ & 293 \\
CRH & $8.06(6.68)$ & $7.70(6.71)$ & 371 \\
Cohen's d & .03 & .09 & \\
\hline
\end{tabular}




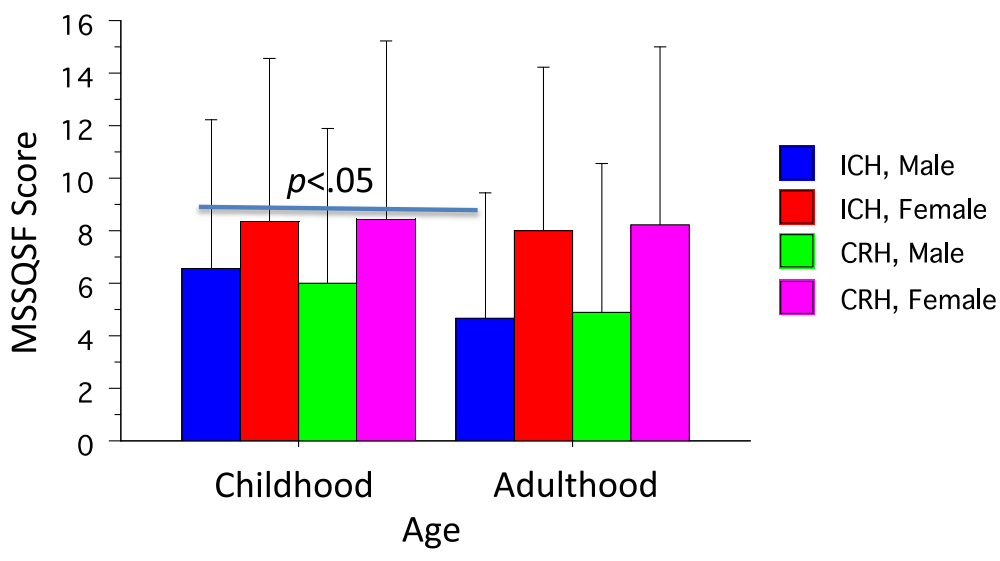

Fig. 1 MSSQSF mean and sd as a function of Handedness, Gender, and Age. Men reported significantly more motion sickness in childhood, compared to adulthood. Women reported significantly more motion sickness than men at both Ages. No effect of Handedness was found

$(\mathrm{F}(1,1)=19.25, p<.0001$, which interacted $(\mathrm{F}(1,660)=$ $10.10, p<.001$. Men experienced motion sickness significantly less in adulthood, compared to childhood (t(137) $=5.77, p<.0001)$. Women did not change in motion sickness as a function of Age, and were significantly more motion sick than men for both Ages $(\mathrm{t}(662)=3.35$ Childhood; $\mathrm{t}(662)=5.67$ Adulthood, $p<.001$ for both comparisons; see Fig. 1). There were no main effects or interactions with Handedness, and effect sizes for these comparisons were small (See Table 1).

\section{Discussion}

Individual differences in Handedness were not related to self-reported motion sickness. This is surprising, given the well-documented differences between handedness groups in neuroanatomy (e.g.: [7]), neurophysiology [13], cognition, and behavior (see [12]). One reason for these findings may involve episodic memory. The $\mathrm{ICH}$ demonstrate superior episodic memory compared to $\mathrm{CRH}$ (e.g.: [13], see also [12], for review); it is possible that results here reflect not actual motion sickness, but differences in recall. For example, $\mathrm{ICH}$ may tend to accurately recall instances of motion sickness, while $\mathrm{CRH}$ may underestimate such experiences. Alternatively, CRH may be more likely than $\mathrm{ICH}$ to falsely recall information (e.g. [3]), and may have over-estimated their motion sickness. Such recall biases would differentially influence handedness effects in self-reported motion sickness. Future work could replicate the present study with a larger sample, or other populations, to examine generalizability.

Future research could directly examine laboratoryinduced motion sickness to resolve whether or not, in actuality, individual differences in handedness are related to motion sickness propensity. Such a study would be particularly interesting in light of work examining gender effects on motion sickness. Specifically, while previous work has reported increased self-reported motion sickness in women, relative to men [11], there were no gender effects in optokinetic-induced motion sickness experience. Interestingly, here, men reported being less motion sick in adulthood compared to childhood, while women reported similar motion sickness in each age. One possible explanation is that women may be more likely to 'admit' to instances of being motion sick, with men being more likely to deny such experiences, particularly in adulthood [2]. Finally, the replication here of differences between men and women in self-reported motion sickness propensity argues for the validity of the measures used, and supports the lack of handedness effects.

\section{Conclusion}

Individual differences in handedness were not related to self-reported motion sickness propensity in either childhood or adulthood. Results may reflect individual differences in handedness effects on memory. Future research could examine a larger population, a different participant group, or actual induction of motion sickness to investigate any relationships further. Men were less likely than women to report motion sickness, and were less motion sick in adulthood relative to childhood. Replication of differences between men and women in self-reported motion sickness propensity argues for the validity of the measures used, and supports the lack of handedness effects. Given the handedness groups demonstrate differences in brain organization, null effects here may shed light on the neural mechanisms that are involved in motion sickness.

\section{Abbreviations}

ANOVA: Analyses of variance; CLH: Consistent-left-handed, those scoring -85 and below on the EHI; $\mathrm{CRH}$ : Consistent-right-handed, those scoring +85 and above on the EHI; EHI: Edinburgh handedness inventory [13];

ICH: Inconsistent-handed, those scoring between +85 and -85 on the EHI MSSQF: Motion sickness susceptibility questionnaire - short form [6];

SD: Standard deviation 


\section{Acknowledgements}

Not applicable.

\section{Funding}

This work was not supported by any funding.

\section{Availability of data and materials}

Datasets are available upon reasonable request.

\section{Authors' contributions}

REP, FB, and KS conceptualized the research and design; REP analyzed the data and wrote the document, along with discussing relevant research with FB; LW programmed Qualtrics, downloaded the data, and formatted the data. All authors read and approved the final manuscript.

\section{Authors' information}

Frederick Bonato is now Provost and Vice-President for Academic Affairs at Saint Peter's University.

\section{Ethics approval and consent to participate}

The research was approved by the Montclair State University IRB, protocol \#IRB-FY16-17-362, and procedures were in accord with the Declaration of Helsinki and all subsequent additions and alteration. All participants provided informed consent via the anonymous, online, consent form.

\section{Consent for publication}

Not applicable.

\section{Competing interests}

The authors declare they have no competing interests.

\section{Publisher's Note}

Springer Nature remains neutral with regard to jurisdictional claims in published maps and institutional affiliations.

Received: 1 January 2018 Accepted: 7 February 2018

Published online: 26 February 2018

\section{References}

1. Arshad Q, Nigmatullina Y, Bronstein AM. Handedness-related cortical modulation of the vestibular-ocular reflex. J Neurosci. 2013;33:3221-7.

2. Cheung B, Hofer K. Lack of gender difference in motion sickness induced by vestibular coriolis cross-coupling. J Vestib Res. 2003;12:191-200.

3. Christman SD, Propper RE, Dion A. Increased interhemispheric interaction is associated with decreased false memories in a verbal converging semantic associates paradigm. Brain Cogn. 2004;56:313-9.

4. Dieterich M, Bense S, Lutz S, Drzezga A, Stephan T, Bartenstein P, Brandt T. Dominance for vestibular cortical function in the non-dominant hemisphere. Cereb Cortex. 2003;13:994-1007.

5. Golding JF. Predicting individual differences in motion sickness susceptibility by questionnaire. Personal Individ Differ. 2006;41:237-48.

6. Hellige JB. Hemispheric asymmetry: What's right and What's left. Cambridge, MA: Harvard University Press; 2001.

7. Luders E, Cherbuin N, Thompson PM, Gutman B, Anstey KJ, Sachdev P, et al. When more is less: associations between corpus callosum size and handedness lateralization. Neurolmage. 2010;52:43-9.

8. Mirabile C, Teicher M. Hand preference, susceptibility to motion sickness, and differential vulnerability to psychiatric admission. Percept Mot Skills. 2002;95:813-4

9. Nooij SA, Vanspauwen R, Bos JE, Wuyts FL. A re-investigation of the role of utricular asymmetries in space motion sickness. J Vestib Res. 2011;21:141-51.

10. Oldfield RC. The assessment and analysis of handedness: the Edinburgh inventory. Neuropsychologia. 1971;9:97-113.

11. Park AH, Hu S. Gender differences in motion sickness history and susceptibility to optokinetic rotation-induced motion sickness. Aviation Space Environmenta Med. 1999;70:1077-80.

12. Prichard E, Propper RE, Christman SD. Degree of handedness, but not direction, is a systematic predictor of cognitive performance. Frontiers in Cognition. 2013;4, Article 9 https://doi.org/10.3389/fpsyg.2013.00009.
13. Propper RE, Patel N, Christman SD, Carlei C. Superior episodic memory in inconsistent-handers: a replication and extension using fNIRS. Memory. 2017;31:1-6.

14. Reason JT, Brand JJ. Motion sickness. Oxford: England: Academic Press; 1975.

15. Richardson AJ. Handedness and visual-motion sensitivity in adult dyslexics. The Irish J of Psychol. 1995;16:229-47.

\section{Submit your next manuscript to BioMed Central and we will help you at every step:}

- We accept pre-submission inquiries

- Our selector tool helps you to find the most relevant journal

- We provide round the clock customer support

- Convenient online submission

- Thorough peer review

- Inclusion in PubMed and all major indexing services

- Maximum visibility for your research

Submit your manuscript at www.biomedcentral.com/submit 